\title{
¿Ética, bioética o ética médica?
}

\author{
ALBERTO ROJAS O.* y LIBIA LARA C.**
}

Ethics, bioethics or medical ethics?

A few years ago, words like Ethics, Bioethics or Medical Ethics were unheard of in clinical medicine, as well as in experimental sciences. However, the increasing number of ethical conflicts in medical practice and dilemmas in clinical research has produced misunderstandings in the use of these terms. Therefore, it presently seems interesting to review the basis of each one of these concepts, as well as the meaning and proper use of them.

Key words: Ethics, Bioethics, Medical Ethics.

\section{Resumen}

Hace pocos años, términos como ética, bioética o ética médica eran ajenos en el uso en medicina clínica, así como en la ciencia experimental. Sin embargo, el aumento progresivo de los problemas éticos en la práctica de la medicina, que en ocasiones son verdaderos dilemas, ha producido confusión en el significado de cada uno de estos conceptos. Es por ello que nos ha parecido de interés revisar estos conceptos, así como el significado y utilidad que tiene cada uno de ellos.

Palabras clave: Ética, Bioética, Ética Médica.

\section{Introducción}

Hace pocos años, términos tales como ética médica, bioética o comité de ética eran ajenos al contexto del ejercicio de la medicina clínica y más aún al de la ciencia experimental. La irrupción de la tecnología como una expresión de progreso, el advenimiento de la computación, que facilitó en gran medida el acceso al conocimiento, así como cambios en el comportamiento de la sociedad, hicieron evidentes algunos procesos adaptativos en la medicina. Merece destacarse, entre otros, una evolución en la relación médico paciente, que pasó de un paternalismo clásico a una relación gobernada por la autonomía del enfermo y la omnipresencia del denominado consentimiento informado. No puede negarse en este sentido el impacto que produjeron los informes de la Comisión Belmont en $1978^{1}$ y un año más tarde, la publicación del trabajo de los bioeticistas T. L. Beauchamp y J. F. Childress ${ }^{2}$, que influyeron radicalmente en el ejercicio de la medicina con la aplicación de los postulados acerca de los cuatro principios bioéticos: autonomía, no maleficencia, beneficencia y justicia, situación que está plenamente vigente hasta nuestros días. En síntesis, el respeto a la autonomía se refiere a que el paciente actúa con conocimiento, intencionadamente, sin influencias externas, la no maleficencia se refiere al hecho de no hacer o provocar daño intencionadamente hacia el enfermo, la beneficencia supone la obligación moral de actuar en beneficio de los otros y, finalmente, la justicia se refiere a que las personas tienen derechos iguales, es decir, deben ser tratadas con igualdad. También este principio aplica a la distribución equitativa de los derechos y responsabilidades o cargas en la sociedad, tomando allí el nombre de justicia distributiva. Sin embargo, una consecuencia no deseada de estos tiempos ha sido la aparición de diferentes modos de judicialización de la medicina. Otro aspecto, negativo a nuestro juicio, que ha surgido con fuerza, es el excesivo tecnicismo que ha caracterizado el ejercicio de la profesión. Ambas situaciones han cooperado, sin duda, para poner a prueba la antigua relación de

\footnotetext{
* Neumólogo.

** Psicóloga. Facultad de Medicina, Universidad Nacional Andrés Bello UNAB, sede Viña del Mar.
} 
confianza en la figura y humanidad del médico. Lamentablemente, puede sostenerse que este fenómeno cruza transversalmente a toda expresión de la medicina.

Como contrapartida a lo que estamos diciendo, se ha revalorizado el papel de los comités de ética, asistenciales o de investigación, como una forma de cooperación en el análisis de los dilemas que surgen de la práctica médica diaria, debido a complejidades cada vez mayores. Los comités son herederos de un proceso evolutivo que se inicia con el juicio de Núremberg, llegando hasta las actuales normas CIOMS -Consejo de Organizaciones Internacionales de las Ciencias Médicas- acerca de investigación en seres humanos ${ }^{3}$.

Sin embargo, en la actualidad, términos como comité de ética, bioética, ética médica, problema ético o dilema ético clínico, a menudo se utilizan en forma indistinta, cuando en realidad su significado apunta a situaciones completamente diferentes. Por tanto, nos ha parecido de interés clarificar estos términos, que no siempre se vinculan al mismo fenómeno en la práctica médica habitual. Es llamativo y quizás algo paradojal, que conceptos surgidos para apoyar la gestión médica, puedan en un primer momento confundir más la situación que pretenden aliviar. Esto se debe a que no siempre existe conocimiento del exacto rol de esta nueva terminología.

A nuestro juicio, no existe hoy ningún centro de atención médica, tanto de pacientes ambulatorios, así como hospitalizados, independientemente de su nivel de complejidad, que no se vea sometido a encrucijadas ético clínicas derivadas de sus propias funciones asistenciales. Pero el fenómeno es más complejo aún, ya que los problemas a los que nos referimos no sólo afectan al binomio médico-paciente, sino que su dificultad se ha extendido también a la relación médico-familiares y con no poca frecuencia a la no siempre fácil relación entre los propios colegas y personal de la salud. Nos atrevemos a plantear que la problemática bioética se ha ido instalando en forma transversal en la atención de la salud y ya no sólo es un asunto que compete a los médicos y sus pacientes, sino que hoy puede decirse que los aspectos éticos se vinculan ampliamente, desde la relación básica entre el médico y su paciente hasta la misma administración de la salud, donde aspectos como la justicia distributiva, también plantean con más frecuencia de la deseada, serios escollos a resolver. Para poder aclarar todo este conjunto de conceptos, vamos a tratar de explicarlos de forma concisa, con la finalidad de aplicarlos adecuadamente a cada situación.

\section{Bioética}

La Bioética es una disciplina reciente, que nació en el seno de la cultura norteamericana como respuesta a la necesidad social y profesional de encontrar una solución para los nuevos dilemas éticos que surgían a causa del avance tecnológico. De acuerdo con la definición otorgada por la Enciclopedia de Bioética, la entenderemos como "estudio sistemático de la conducta humana en el área de las ciencias de la vida y el cuidado de la salud, en cuanto que dicha conducta es examinada a la luz de los valores y principios morales". Naturalmente, esta definición sólo representa uno de los aspectos abarcados por ella, puesto que bioética es una palabra transversal e interdisciplinar, que comprende gran parte de disciplinas científicas y en la cual intervienen otras como la medicina, ciencias biosanitarias, derecho, política, economía, filosofía, biología, psicología, antropología, sociología, bioquímica, estadística, ingeniería y sobretodo, ética. Cada una de estas ciencias aporta principios y observaciones que entran a formar parte de este vasto conjunto multidisciplinar que constituye hoy la bioética.

Sin embargo, lo que Van Rensselaer sugirió en $1971^{5}$ con la palabra bioética fue la unión del mundo de los hechos, de la ciencia, y el mundo de valores, y en particular de la ética, por considerar que se desplazaban por caminos divergentes. Una aclaración necesaria en este punto, es que el trabajo de Van Rensselaer surgió desde el ámbito de la medicina, más acotado que el concepto de bioética global, aceptada ésta como una ética de la vida pero entendida en lato sensu, y que apunta a los actos del hombre sobre la vida humana, así como animal y medioambiental. Sin embargo, ha sido la medicina, la que ha contribuido en forma decisiva al surgimiento $\mathrm{y}$ aplicación práctica de la bioética y a su posterior expansión y sobre todo, justificación, en el ámbito del hombre enfermo.

\section{Ética Médica}

En cuanto a la ética médica y su fundamento, proviene en gran parte desde la propia actividad de los médicos, ya que a diario enfrentan problemas clínicos. La solución de ellos dependerá, en gran medida de conocimientos y habilidades que han adquirido a lo largo de la formación y experiencia. Sin embargo, estos problemas clínicos, a menudo, vienen acompañados de dilemas éticos, para los cuales no existe una simple respuesta y cuya complejidad va más allá de normas o tecnicismos. 
En el último período, la medicina ha sufrido un cambio importante. La postura tanto del médico como del paciente y todo lo que conlleva dicho proceso en la relación médico-paciente, se ha permutado. El paciente ha pasado de tener una postura pasiva a una postura activa, en la cual toma cada vez más decisiones en todos los procesos que le involucran. El médico debe mantener informado de toda la situación al paciente. Pero no es suficiente. Se debe cumplir con el requisito dinámico, tanto verbal como escrito del consentimiento informado, que parece presidir todo acto médico en la actualidad. En este contexto, se debe respetar la autonomía del paciente, en todo momento, con mayor fuerza si el enfermo ha comprendido la situación que lo afecta, sin dejar de lado la figura del representante legal o persona que está a cargo del enfermo.

La ética médica, se ocupa por lo tanto de asuntos de orden práctico, relacionados con la ciencia médica, entendida como la atención de enfermos y los problemas que se pudieran derivar de ello, pero además de una serie de otras situaciones de diferente complejidad, donde suele darse intenso debate, como por ejemplo, reproducción asistida, eutanasia, prolongación de la muerte, trasplantes de órganos, aborto, orden de no reanimación, retirada de tratamientos, adecuación terapéutica, etc. Es aquí donde se produce la tensión entre el mundo de los hechos, avances tecnológicos y datos concretos, respecto del mundo de los valores y principios morales. La ética, a secas, el "ethos" de la cultura griega, como parte de la filosofía, aporta la necesaria capacidad de reflexión y deliberación que requieren los problemas de la medicina, cuando son sobrepasados por el mero dato o la norma.

Muchas veces son los mismos progresos tecnológicos que el hombre ha sido capaz de crear, los que justifican a nuestro entender, poner unos límites en temas que tienen relación con la medicina, cuyo fin último no es otro que proteger en todo momento el buen actuar médico y garantizarle al paciente la justicia de la cual es merecedor.

\section{Problema ético clínico}

Muchas veces el equipo médico enfrenta situaciones que no son fáciles de resolver. La escasez de recursos, altos costos de la tecnología, quién merece más una cama en cuidados intensivos, reanimar o no a un recién nacido gravemente enfermo, comunicar un diagnóstico ominoso al paciente o su familia, son apenas unos pocos ejemplos de la dificultad a la que nos referimos. En el caso de la clínica, es aquí donde la reflexión ética y el conocimiento bioético general se tornan indispensables para zanjar estos dilemas. Este es el lugar preciso que ocupa el comité de ética asistencial del hospital. De constitución interdisciplinar, autónomos, son en la actualidad imprescindibles a la hora de dar apoyo al trabajo del médico y aportar con la experiencia de sus miembros. No tienen el objetivo de sancionar ni juzgar, sino que, a solicitud expresa de los médicos o directivos del hospital, deben elaborar una respuesta que tendrá, siempre, el carácter de sugerencia. Existen otras funciones de los comités de ética, tanto asistenciales como de investigación, algunas de ellas han sido analizadas en publicaciones previas ${ }^{6-8}$.

Finalmente, se puede decir que, los profesionales de la salud, para poder solucionar los problemas éticos que se presentan en su labor, necesitan trascender la ciencia experimental, acudir al propio bagaje cultural y a la ayuda de otras disciplinas, como la filosofía, metafísica, ética, psicología, derecho, para encontrar respuesta a las situaciones de incertidumbre que se presentan en el quehacer diario.

\section{Conclusión}

La palabra ética, del griego "ethos", es uno de los pilares de la filosofía. Apunta al comportamiento moral de la persona en el mundo. Puede decirse que, en un modo amplio, resuelve la inquietud respecto a qué es lo bueno a hacer, y lo malo a evitar. En otro sentido, la bioética se vincula, como una parte de la ética, con un concepto más acotado hacia la vida en el medio ambiente, desde un punto de vista general, tanto humana, vegetal y animal.

En cambio, la ética médica une las normas de comportamiento adecuado, bueno o correcto, en el campo de la atención al hombre enfermo, como ente concreto. Es aquí donde los conceptos de ética y bioética adquieren un propósito palpable y, por tanto, su adecuado conocimiento, aplicación y juicio de valor son relevantes para el ejercicio de la medicina en nuestros tiempos. Conviene aclarar que lo que habitualmente se nombra como bioética, en la atención de los enfermos y su contexto global en los dilemas de la salud, en realidad se identifica mejor con la ética médica, que le es más propia porque aplica principios precisos de lo que puede entenderse como una moral médica. Esta última, entendida como una deliberación seria, argumentada, razonada y razonable. 


\section{Bibliografía}

1.- PRINCIPIOS Y GUÍAS ÉTICOS PARA LA PROTECCIÓN DE LOS SUJETOS HUMANOS DE INVESTIGACIÓN. Comisión Nacional para la protección de los sujetos humanos de investigación biomédica y del comportamiento. Departamento de Salud, Educación y Bienestar de los Estados Unidos de Norteamérica. 18 de abril de 1979. Disponible en: www medicina.uc.cl/ docman/358/doc Consultado: 17 de mayo de 2014.

2.- BEAUCHAMP T L, CHILDRESS J F. Principles of Biomedical Ethics. $6^{\text {a }}$ ed. New York: Oxford University Press, 2009.

3.- CIOMS: Pautas éticas internacionales para la investigación biomédica en seres humanos. Ginebra, 2002 (Traducción por el Programa Regional de Bioética OPS/
OMS, Santiago de Chile, 2003).

4.- SERRANO RUIZ J M. Cuestiones de bioética, Editorial Speiro, Madrid, 1992, págs. 18-9.

5.- VAN RENSSELAER P. Bioethics, Bridge to the future. Prentice-Hall. New Jersey 1971.

6.- LAVADOS M, SALAS S P. Problemas éticos en los proyectos de investigación biomédica presentados al Comité de Ética de la Escuela de Medicina de la Pontificia Universidad Católica de Chile. Rev Med Chile 1997; 125: 1011-8.

7.- SOTOMAYOR M A. Algunas consideraciones en torno a la bioética y a la investigación científica biomédica. Rev Chil Enf Respir 2007; 23: 7-10.

8.- GAUDLITZ M. Reflexiones sobre los principios éticos en investigación biomédica en seres humanos. Rev Chil Enf Respir 2008; 24: 138-42.
Correspondencia a:

Dr. Alberto Rojas Osorio

Facultad de Medicina, sede Viña del Mar

Universidad Nacional Andrés Bello

Email: arojas1040@yahoo.com 